\title{
Zur Frage von der Ende 1889 auf mehreren Sternwarten beobachteten Veränderung der geographischen Breite.

\author{
Von M. Nyrén.
}

Da ich im vergangenen Herbst erst gegen Ende October von einer längeren ausländischen Reise zurückkehrte und der Himmel dann bis zum Ende des Jahres bei uns fast unaufhörlich bewölkt war, so haben in dieser Zeit nur einige wenige Beobachtungen des Polarsterns mit dem Verticalkreis gewonnen werden können. $\mathrm{Da}$ aber jeder Beitrag zu der von Professor Helmert in A. N. 2963 mitgetheilten Wahrnehmung, wonach man Ende vorigen Jahres in Berlin, Potsdam, Prag und Strassburg bedeutende Verkleinerungen der Breite beobachtet, seine Bedeutung hat, so erlaube ich mir die aus diesen paar Messungen folgenden Werthe für die Polhöhe unseres Verticalkreises hier mitzutheilen.

Aus $6_{3}$ in beiden Culminationen angestellten Beob. achtungen von $\&$ Urs. min. in der Zeit März bis Juni 1889 folgt :

$$
\varphi=59^{\circ} 46^{\prime} \times 8^{\prime \prime} .40
$$

Corr. der Decl. von $\alpha$ Urs. min. in B. J. $=-0$ " 105 .

Aus 20 eben solchen Beobachtungen im März und April dieses Jahres bekomme ich:

$$
\varphi=59^{\circ} 46^{\circ} \text { I } 8^{\prime \prime} 33
$$

Corr. der Decl. von $\alpha$ Urs. min. in B. J. $=-0$ " 102 .

Nehmen wir dann für die Decl. des B. J. von $\alpha$ Urs. min. als Correction -o"' o, so geben die erwähnten Beobachtungen vom vergangenen Herbst:

$$
\begin{array}{cr}
\text { I889 Nov. } 20 & \varphi=59^{\circ} 46^{\prime} 18.76 \\
>20 \text { s.p. } & 18.56 \\
\text { Dec. } 18 & 18.35 \\
>29 & 18.02
\end{array}
$$

Als w. F. einer Beobachtung, verglichen mit dem Jahresmittel, habe ich bei einer früheren Untersuchung \pm 0 " 136 gefunden.

Um zu zeigen, inwiefern die Unterschiede dieser Resultate der Refraction zugeschrieben werden können, erlaube ich mir noch folgende an denselben Tagen angestellte Beobachtungen mitzutheilen. Die zu Grunde liegenden
Sterndeclinationen habe ich mit Ausnahme von $\beta$ und o Ceti, die dem B. J. entlehnt sind, aus einigen neueren Beobachtungen am selben Instrument abgeleitet:

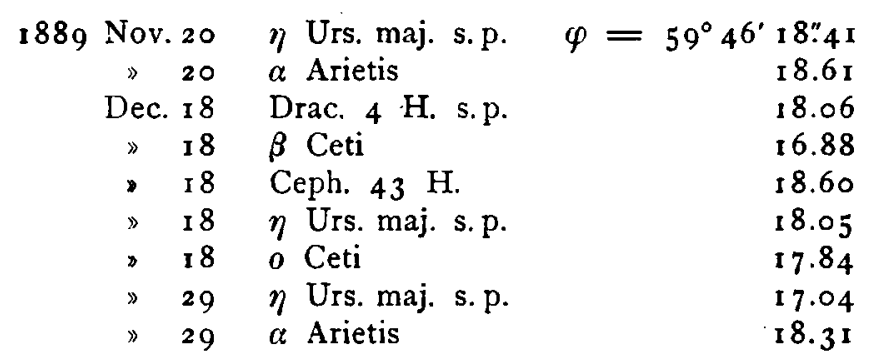

Wie man sieht, deuten auch diese Beobachtungen auf eine in der Zwischenzeit vor sich gegangene Verkleinerung der Polhöhe. Da sie aber zu beiden Seiten des Zeniths angestellt sind, so könnten ja solche Veränderungen nicht anders durch die Refraction erklärt werden, als durch die Annahme zufälliger excentrischer Lagerung der Luftschichten gleicher Dichtigkeit.

Obgleich nun die Beobachtungen sowohl des Polarsterns wie der anderen Sterne eine messbare Verkleinerung der Polhöhe in dem erwähnten Zeitraum andeuten, so würde ich doch, mit Rücksicht auf die geringe Zahl meiner Beobachtungen, diesen Resultaten allein wenig Bedeutung beilegen. In Verbindung mit den von Helmert mitgetheilten Wahrnehmungen scheinen sie aber etwas Beachtung zu verdienen.

In diesem Frühjahr habe ich regelmässige Beobachtungen einiger hellen Zenithsterne mit dem grossen Passageninstrument im ersten Vertical in Gang gesetzt. Die sehr schön übereinstimmenden Reihen, welche der Beobachter, Herr Candidat Wanach, schon damit gewonnen, lassen die Hoffnung als berechtigt erscheinen, dass mit Hülfe gleichzeitiger Beobachtungen an den beiden ge. nannten Instrumenten das berührte Problem, welches mich schon seit 20 Jahren lebhaft interessirt und beschäftigt hat, in Bezug auf Pulkowa innerhalb nicht zu langer Zeit gelöst werden kann.

Pulkowa 1890 Juni.

M. Nyrén.

\section{Elementi ellittici ed osservazioni di (264) Libussa in quarta opposizione.}

Prendendo in conto le tre opposizioni trascorse del pianetino (264) Libussa e le perturbazioni da dicembre I 886 a maggio I 889 per opera di Giove e di Saturno ottenni gli elementi corretti seguenti:

Epoca ed osculazione 1889 Aprile 20.5 t. m. Berlino.

$$
\begin{aligned}
& M=190^{\circ} \text { I } 7^{\prime} \text { 1 } 6^{\prime \prime} 6 \\
& \omega=33^{6} 53 \quad 3.9 \\
& \delta=\begin{array}{lrr}
50 & 5 & 13.6
\end{array} \quad \text { M. equ. } 1890.0 \\
& i=102719.9 \\
& \varphi=7^{\circ} 53^{\circ} 4^{\prime \prime} .7 \\
& \mu=75^{8.0493^{\circ}} \\
& \log a=0.446873
\end{aligned}
$$

Il pianeta fu da me ritrovato in quarta opposizione quantunque in declinazione $-36^{\circ}$ e di $12^{\mathrm{ma}}$ grandezza. 